\title{
Criminologie
}

\section{Le rôle des facteurs développementaux dans la détermination de la responsabilité morale chez les jeunes : une étude pilote évaluant les opinions d'experts légaux et cliniques}

\author{
Irina Demacheva, Noémie Aubert Bonn, Sébastien Lucero, Martin Ladouceur \\ et Amir Raz
}

Volume 45, numéro 2, automne 2012

URI : https://id.erudit.org/iderudit/1013725ar

DOI : https://doi.org/10.7202/1013725ar

Aller au sommaire du numéro

Éditeur(s)

Les Presses de l’Université de Montréal

ISSN

0316-0041 (imprimé)

1492-1367 (numérique)

Découvrir la revue

Citer cet article

Demacheva, I., Aubert Bonn, N., Lucero, S., Ladouceur, M. \& Raz, A. (2012). Le rôle des facteurs développementaux dans la détermination de la responsabilité morale chez les jeunes : une étude pilote évaluant les opinions d'experts légaux et cliniques. Criminologie, 45(2), 187-218. https://doi.org/10.7202/1013725ar

\section{Résumé de l'article}

Récemment, la recherche sur le développement cognitif fait part d'une grande source d'information concernant la responsabilité morale attribuable aux délinquants juvéniles. Cependant, l'intégration de ces connaissances dans le milieu légal demeure largement théorique. En utilisant un sondage en ligne, nous avons évalué les opinions, les croyances et les attitudes d'experts légaux et cliniques à propos de l'impact des facteurs développementaux sur la responsabilité morale des jeunes. Nos résultats suggèrent que la communauté légale est modérément consciente de la recherche émergente en science développementale portant sur la responsabilité morale des jeunes. Or le fossé entre la théorie et la pratique persiste. En ce qui a trait à la responsabilité morale des adolescents et leur capacité à subir un procès, par exemple, les policiers attribuent significativement moins d'importance aux facteurs développementaux que ne le font les experts cliniques. Nous soulignons le besoin d'un dialogue plus rapproché entre le droit et la science développementale pour favoriser la création d'un consensus et pour améliorer le traitement des jeunes délinquants. 


\section{Le rôle des facteurs développementaux dans la détermination de la responsabilité morale chez les jeunes: une étude pilote évaluant les opinions d'experts légaux et cliniques}

Irina Demacheva

Département de psychiatrie, Université McGill

irina.demacheva@gmail.com

Noémie Aubert Bonn

Département de psychiatrie, Université McGill noemie.aubertbonn@mail.mcgill.ca

\section{Sébastien Lucero}

Département de psychologie, Université McGill sebastienlucero@gmail.com

Martin Ladouceur

Département de génétique humaine, Université McGill

mladouceur@epimgh.mcgill.ca

Amir Raz

Département de psychiatrie, Université McGill

amir.raz@mcgill.ca

Traduit de l'anglais par Rabia Mzouji

RÉSUMÉ • Récemment, la recherche sur le développement cognitif fait part d'une grande source d'information concernant la responsabilité morale attribuable aux délinquants juvéniles. Cependant, l'intégration de ces connaissances dans le milieu légal demeure largement théorique. En utilisant un sondage en ligne, nous avons évalué les opinions, les croyances et les attitudes d'experts légaux et cliniques à propos de l'impact des facteurs développementaux sur la responsabilité morale des jeunes. Nos résultats suggèrent que la communauté légale est modérément consciente de la recherche émergente en science développementale portant sur la responsabilité morale des jeunes. Or le fossé entre la théorie et la pratique persiste. En ce qui a trait à la responsabilité morale des adolescents et leur capacité à subir un procès, par exemple, les policiers 
attribuent significativement moins d'importance aux facteurs développementaux que ne le font les experts cliniques. Nous soulignons le besoin d'un dialogue plus rapproché entre le droit et la science développementale pour favoriser la création d'un consensus et pour améliorer le traitement des jeunes délinquants.

MOTS-CLÉS • Développement cérébral, adolescence, aptitude à subir un procès, système de justice pénale pour les adolescents

\section{Introduction $^{1}$}

La recherche récente sur le développement du cerveau, notamment celle qui concerne les adolescents, offre de nouvelles perspectives sur des problèmes judiciaires concernant la responsabilité morale des jeunes (Maroney, 2009). Ces résultats vont de pair avec des décisions récentes des cours suprêmes des États-Unis (Graham c. Florida, 2012) et du Canada (Roper c. Simmons, 2005 ; R. c. D.B., 2008). Le sens global de ces résultats et de ces décisions judiciaires fait appel à une réévaluation de la responsabilité morale (blameworthiness) des jeunes (Scott et Steinberg, 2008). L'intégration des découvertes de la recherche développementale aux affaires judiciaires, cependant, a été largement inefficace (Maroney, 2009). Nous examinerons dans cet article les connaissances, les croyances et les attitudes de professionnels légaux et cliniques en ce qui concerne le problème de la responsabilité morale des jeunes. Nous cernerons aussi comment un dialogue plus rapproché entre le droit et la science pourrait paver la voie au consensus et à un meilleur traitement des jeunes délinquants.

Les adolescents présentent un risque plus élevé de conduites criminelles (Agnew, 2003; Kambam et Thompson, 2009) - c'est-à-dire violer la loi, ce qui peut entraîner des pénalités telles que l'incarcération ou l'amende. Des recherches montrent que les taux d'arrestation atteignent des sommets entre les âges de 15 à 18 ans (Gottfredson et Hirschi, 1990). Les comportements à risque et la tendance à abuser de drogues ou d'alcool augmentent souvent, de plus, alors que des proces-

1. Nous remercions Claire Champigny pour les commentaires qu'elle a apportés aux premiers brouillons de ce manuscrit. De plus, nous tenons à souligner l'aide des membres du personnel de l'Université McGill pour leur soutien technique et l'entretien du sondage en ligne, plus spécifiquement Daniel Schwartz du IT Customer Services [Service à la clientèle du centre de technologies informatiques] et Maggie Lattuca des Educational Technologies [Technologies de l'enseignement]. Le Dr Raz remercie l'appui financier de la Chaire de recherche du Canada ainsi que le grand appui des Instituts de recherche en santé du Canada et du Conseil de recherches en sciences naturelles et en génie du Canada. 
sus de maturation sont en cours (Spear, 2000). Alors que les aires visuelles et auditives du cerveau ont une apparence semblable à celle des adultes dès la fin de la période préscolaire (Shonkoff et Phillips, 2000), les lobes frontaux, qui sont des aires cérébrales clés impliquées dans les fonctions exécutives telles que la planification, l'attention et le contrôle, peuvent atteindre leur pleine maturation seulement vers le début de l'âge adulte (Giedd et al., 1999; Sowell et al., 1999; Blakemore et Choudhury, 2006; Yurgelun-Todd, 2007). C'est dans ce contexte que onze éminents neuroscientifiques en science développementale ont adressé une lettre au Tribunal dans laquelle ils appuient l'ébauche d'un livre blanc qui soutient que le cerveau de l'adolescent, comparativement à celui de l'adulte, est immature en ce qui concerne les fonctions qui contribuent au contrôle exécutif du comportement, ces caractéristiques entravent la capacité des jeunes à prendre des décisions (Luna et al., 2009), ce qui contribue à leur tendance à s'engager dans des conduites criminelles (Steinberg et Scott, 2003).

La capacité à prendre des décisions, soit la capacité d'évaluer correctement les conséquences à la fois positives et négatives d'une action (Kambam et Thompson, 2009) est déficiente chez les adolescents, ce qui constitue un argument au soutien de leur responsabilité morale réduite (Cauffman et Steinberg, 2000b). Par exemple, les adolescents utilisent un système d'évaluation des risques et des récompenses dans lequel moins de poids est attribué au risque qu'à la récompense (Steinberg et Scott, 2003), qui a pour conséquence une plus grande tendance à s'engager dans des comportements à risque (Gardner et Steinberg, 2005; Magar et al., 2008). Le développement cognitif comprend deux composantes importantes de la prise de décisions: la comprébension, soit la capacité de comprendre les informations qui ont trait à une décision, et le raisonnement, soit l'habilité d'utiliser cette information de manière logique pour faire un choix (Diamond, 2006). En ce sens, le développement d'habiletés cognitives comme l'attention (Raz et Buhle, 2006) peut améliorer les conduites auto-régulantes dont la prise de décision (Diamond, 2006). Alors que la capacité de comprendre et de s'engager dans un raisonnement logique ressemble à celle de l'adulte dès la mi-adolescence, soit vers l'âge de 15 ou 16 ans (Keating, 2004), les habilités de prise de décisions demeurent pauvres en raison d'une maturation psychosociale incomplète (Scott et Steinberg, 2008). La maturation psychosociale déficiente des jeunes, dont leur incapacité à fonctionner de manière indépendante et celle d'interagir adéquate- 
ment avec les autres, (Greenberger et Sørensen, 1974) est caractéristique de la mi-adolescence. Les jeunes sont particulièrement vulnérables face à la pression de leurs pairs (Maxwell, 2002; Steinberg et Scott, 2003; Scott et Steinberg, 2008; Shook et al., 2009), un fort déterminant de la propension aux comportements à risque (Santor et al., 2000; Gardner et Steinberg, 2005). De plus, l'immaturité psychosociale prédit le comportement antisocial des jeunes avec plus de justesse que l'âge (Cauffman et Steinberg, 2000a). Ainsi, tant l'immaturité cognitive que psychosociale des jeunes semble rendre compte de leurs pauvres habiletés de prises de décisions et de leurs comportements criminels, ce qui rend leur responsabilité morale moins importante que celle des adultes (Cauffman et Steinberg, 2000b).

Comparés aux adultes, les adolescents démontrent une moindre aptitude à subir un procès (Scott et Grisso, 2004). Cette aptitude, évaluée de manière semblable aux États-Unis et au Canada, repose sur la capacité de l'accusé à comprendre la nature et les conséquences éventuelles des poursuites ainsi qu'à consulter un avocat (Laberge et al., 1995; Jackson, 2008). De nombreux adolescents ne possèdent pas encore les compétences cognitives requises pour comprendre et participer pleinement au processus judiciaire (Viljoen et Roesch, 2005), et ce, même dans une cour pour mineurs (Viljoen et Grisso, 2007). Suivant cette logique, au Canada, la Loi sur le système de justice pénale pour les adolescents qui fut instaurée en 2003 empêche désormais le transfert de jeunes détenus aux cours criminelles avant qu'ils ne soient jugés coupables (Doob et Cesaroni, 2004). De plus, même lorsqu'une peine d'adulte est appliquée, les juges canadiens doivent obligatoirement considérer l'âge et le potentiel de réhabilitation du jeune afin de choisir une peine adaptée (Bala et Anand, 2009). Contrairement au Canada, où la loi criminelle est exclusivement sous législation juridique du gouvernement fédéral (Caufield, 2001), chaque État aux États-Unis a le pouvoir de mettre en œuvre ses propres lois juridictionnelles dans les procédures de la justice pour les jeunes (Hughes, 1996; Friedman, 2005). Par conséquent, les lois de transfert diffèrent d'un État américain à l'autre. Cependant, malgré cette disparité, la plupart des États facilitent le transfert des jeunes vers les cours criminelles pour adultes. Jusqu'à 22 des États ont au moins une disposition légale selon laquelle l'âge minimum d'un tel transfert n'est pas spécifié (OJJDP, 2011). Les transferts d'adolescents aux cours pour adultes sont donc fréquents aux États-Unis (Redding, 2003; Patapis, 2006). Malheureusement, les cours criminelles pour adultes considèrent 
rarement que l'immaturité des jeunes est un motif de réduction de peine (Carmichael, 2010). En d'autres mots, de nombreux législateurs américains semblent négliger les facteurs développementaux dans le jugement des jeunes délinquants (Freeman et Goodenough, 2009; Fagan, 2010).

Les professionnels du système de justice pénale peuvent avoir recours à un privilège connu sous le nom de discrétion, soit une marge de manœuvre laissée au tribunal qui offre une certaine autonomie aux acteurs juridiques. Certaines décisions juridiques reflètent les évaluations et jugements personnels des juges ou autres acteurs pénaux (Galligan, 1990). Forst et Bushway (2010) soulignent comment l'usage du pouvoir de discrétion par certains acteurs de la justice criminelle peut avoir des conséquences sur les peines. Alors que le pouvoir discrétionnaire des policiers influence les taux d'arrestation, la qualité de ces arrestations ainsi que la discrétion des procureurs ont, quant à elles, un impact sur les taux de condamnation. De plus, le pouvoir discrétionnaire des procureurs à recommander et des juges à imposer des peines affecte le taux et le terme des incarcérations. Cependant, l'influence que peuvent avoir les officiers de la justice criminelle sur les résultats des peines varie d'un pays à l'autre. D'une part, de nombreux États américains n'offrent aucune ou très peu de pouvoir discrétionnaire à leurs juges (Pfaff, 2006). D'autre part, au Canada, les juges bénéficient d'un pouvoir discrétionnaire juridique (Bala et al., 2009) leur permettant d'influencer les résultats des peines (Cesaroni et Bala, 2008). Ce pouvoir a été récemment remis en question par des décisions politiques visant à juger les adolescents comme les adultes et à réduire l'espace discrétionnaire laissé aux juges (Loi sur la sécurité des rues et des communautés, 2011). Cette nouvelle loi n'a cependant pas encore transformé le système judiciaire canadien, et les attitudes des officiers de la justice pénale envers la recherche développementale peuvent avoir un grand impact sur la peine des jeunes.

Le but de cette enquête était de cerner les connaissances, les attitudes, les opinions et les croyances des experts légaux et cliniques en ce qui concerne la science développementale et la justice des jeunes. Nous avons émis l'hypothèse que les experts légaux, comparés aux experts cliniques, attribueraient moins d'importance aux déficits du développement des adolescents. Espérant donner l'exemple, nous visons à susciter un important dialogue entre les experts du droit et de la science, permettant ainsi d'amorcer les démarches vers un consensus dans ce domaine sinon fragmenté et disjoint. 


\section{Méthode}

Le sondage

En utilisant le logiciel libre de sondage en ligne LimeSurvey, nous avons testé l'interface entre la connaissance de la science du développement et les potentielles implications pour la responsabilité morale de la jeunesse et l'aptitude à subir un procès. Afin de nous assurer que les questions du sondage étaient clairement formulées, nous avons testé le sondage auprès de certains experts légaux et cliniques avant de conduire cette étude pilote. Le sondage était composé de quatre questions démographiques et dix questions additionnelles. La plupart des questions étaient à choix multiples (format fermé), mais certaines questions offraient l'option de donner des réponses courtes (format ouvert). Quelques questions comprenaient une échelle de Likert à 5 points. La plupart des questions contenaient une option: «Je ne peux pas répondre.» Le sondage est disponible en ligne sur: http://tinyurl.com/justicejuvenile ainsi que dans l'annexe.

\section{Analyse statistique}

Nous avons fait les analyses statistiques de nos données en utilisant la $10^{\mathrm{e}}$ version du logiciel Stata (StataCorp. 2007. College Station, TX: StataCorp LP). Nous avons examiné et analysé les données en utilisant des statistiques descriptives et des distributions de fréquences. Nous avons utilisé un modèle de régression logistique pour évaluer comment les experts différaient dans leurs opinions; par exemple, quelles sont les différences d'opinions entre les experts cliniques et légaux en ce qui a trait à l'impact des facteurs développementaux sur la responsabilité morale des jeunes et leur aptitude à subir un procès? Nous avons traité l'option «Je ne peux pas répondre» comme donnée manquante et avons appliqué la correction de White (1980) pour tenir compte de l'hétéroscédasticité.

Nous avons traité l'âge, la connaissance du système de justice pénale pour les adolescents (SJPA), le pays et le sexe des experts comme facteurs additionnels pouvant influencer les opinions sur le traitement des délinquants juvéniles. En effet, puisque le débat concernant le traitement des jeunes délinquants en regard des étapes de développement cognitif est relativement récent (Maroney, 2009; Jennings, 2010), il est possible que les experts plus âgés connaissent moins bien la recherche sur le développement. De la même manière, la connaissance du SJPA peut être 
attribuée à une conscience accrue des thèmes entourant ce débat. De plus, la localisation géographique peut influencer la façon dont les experts conçoivent les affaires légales puisque la manière de prendre les décisions de politique publique est propre à chaque pays (Luciana, 2011). Finalement, puisque les femmes ont tendance à être plus empathiques que les hommes (Klauer et Winkeler, 2002; Burleson, 2003), elles seront possiblement plus disposées à penser que les facteurs développementaux affectent la responsabilité morale et l'aptitude à subir un procès.

Dans notre analyse, nous avons transformé les notations de l'échelle de Likert 1-5 ( 1 = pas du tout / 5 = extrêmement) en indices dichotomiques correspondant à des impacts considérés comme faibles $(1,2)$ ou élevés $(3,4,5)$ sur les facteurs en question. Ces transformations nous ont permis de faire la distinction entre les experts qui attribuaient relativement peu d'importance aux facteurs développementaux et ceux qui en attribuaient beaucoup, et d'arriver ainsi à comparer leurs opinions par rapport aux affaires légales concernant des jeunes délinquants.

\section{Participants}

Pour recruter des participants, nous avons contacté de nombreuses associations professionnelles d'experts légaux et cliniques et leur avons demandé de faire circuler notre sondage parmi leurs membres. Nous avons principalement visé des associations basées au Canada et aux États-Unis. Les organisations qui ont fait circuler le sondage incluent l'Association du Barreau canadien, le ministère de la Justice du Canada, American Judges Association [l'Association américaine des juges], International Association of Crime Analysts [Association internationale des analystes en renseignements criminels], American Academy of Child and Adolescent Psychiatry [Académie américaine des psychiatres pour enfants et adolescents], Royal Australian and New Zealand College of Psychiatrists [Collège royal australien et néo-zélandais des psychiatres], et British Columbia Child and Youth Health Research Network [Réseau de la recherche sur les enfants et les adolescents de la ColombieBritannique]. La participation au sondage était anonyme et volontaire, et les participants ne recevaient aucune compensation monétaire.

Deux cent quatre-vingt-onze individus ont répondu au sondage (voir le tableau 1 pour la distribution des répondants par champ d'expertise); quarante-deux pour cent des répondants venaient du Canada, quarantesix pour cent des États-Unis, et douze pour cent du reste du monde 
(ex.: Australie, Nouvelle-Zélande); tous les experts légaux provenaient soit du Canada, soit des États-Unis. Les répondants étaient âgés de 20 à 78 ans (moyenne $[\mathrm{m}]=47,1$; écart-type [é.t.] $=12,2$ ) et constituaient une proportion équivalente d'hommes (48\%) et de femmes (52\%). De ces 222 répondants $76 \%$ ont indiqué qu'ils connaissaient très bien le SJPA (voir le tableau 1 pour la distribution des fréquences de grande connaissance du SJPA par expertise).

TAB LEA U 1

La distribution des participants par expertise

\begin{tabular}{|l|c|c|}
\hline Spécialistes & $\mathrm{n}$ & Fréquence en pourcentage \\
\hline Policier & 29 & $\begin{array}{c}10 \\
(90)\end{array}$ \\
\hline Analyste en renseignements criminels & 28 & 9,6 \\
& & $(68)$ \\
\hline Juge & 35 & 12 \\
& & $(100)$ \\
\hline Avocat & 75 & 25,8 \\
& & $(91)$ \\
\hline Psychiatre & 38 & 13,1 \\
& & $(66)$ \\
\hline Pédiatre & 25 & 8,6 \\
& & $(16)$ \\
\hline Psychologue & 23 & 7,9 \\
& & $(52)$ \\
\hline Travailleur social & 38 & 13,1 \\
& & $(84)$ \\
\hline Total: & 291 & 100 \\
\hline
\end{tabular}

Les nombres entre parenthèses présentent le pourcentage des spécialistes ayant indiqué avoir une grande connaissance du SJPA.

\section{Résultats}

La responsabilité morale des jeunes

Nous avons examiné les différences d'opinions des experts en ce qui concerne l'influence des facteurs développementaux sur la responsabilité morale des jeunes (se référer à la figure 1 pour le pourcentage de distribution des réponses par expertise). Nous avons effectué des régressions logistiques multiples afin d'évaluer le lien entre l'expertise et l'impact dû aux facteurs développementaux (élevé $=1$, faible $=0$ ) en 
utilisant chaque spécialisation comme une catégorie de référence distincte. Le seul groupe d'experts qui différait significativement de certains autres groupes était celui des policiers (voir tableau 2, modèle 1). Ceux-ci étaient moins portés que les avocats $(p<0,05)$, les psychologues $(\mathrm{p}<0,05)$ et les travailleurs sociaux $(\mathrm{p}<0,05)$ à attribuer une importance élevée aux facteurs développementaux quant à la responsabilité morale des jeunes.

FIGURE 1

La distribution des réponses pour la question «Pensez-vous qu'il y ait des traits développementaux ayant une influence sur la responsabilité morale réduite des jeunes?» $(n=291)$

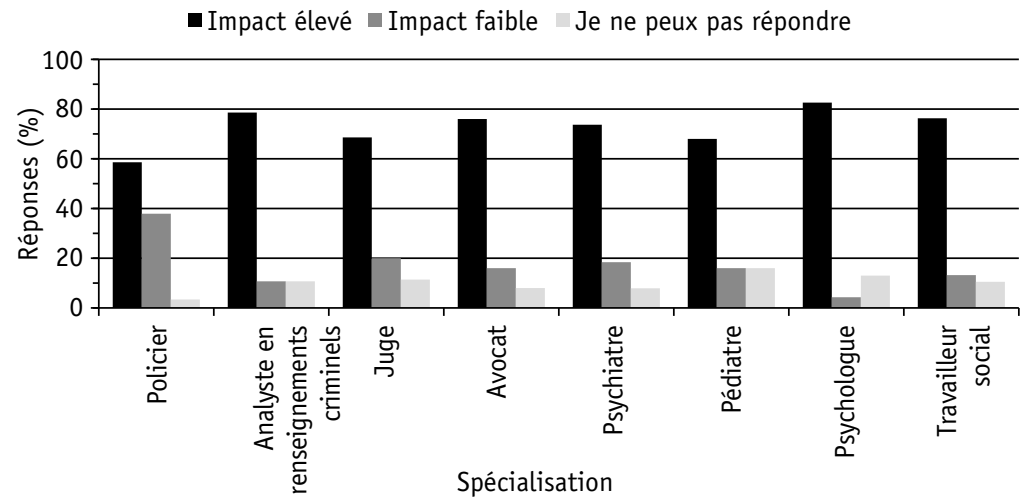

Aptitude des jeunes à subir un procès

Nous avons examiné en quoi les experts différaient dans leur perception de l'influence des facteurs développementaux sur l'aptitude des jeunes à subir un procès (voir la figure 2 pour la distribution en pourcentage des réponses des experts). Nous avons analysé nos données à partir de régressions logistiques multiples pour mieux comprendre le lien entre l'expertise et l'impact des facteurs développementaux (élevé $=1$, faible $=0$ ) en utilisant chaque spécialisation comme une catégorie de référence distincte. Les réponses des psychiatres et des travailleurs sociaux différaient significativement de certaines des autres groupes (voir tableau 2, modèles 2 et 3). Les psychiatres étaient $89 \%, 91 \%$ et $84 \%$ plus susceptibles que, respectivement, les policiers $(p<0,05)$, les analystes en renseignements criminels $(\mathrm{p}<0,05)$ et les juges $(\mathrm{p}<0,05)$, d'attribuer 
TAB LEA U 2

Les résultats provenant des régressions logistiques

\begin{tabular}{|l|c|c|c|c|c|}
\hline & Modèle 1 & Modèle 2 & Modèle 3 & Modèle 4 & Modèle 5 \\
\hline Policier & - & $\begin{array}{c}0,11^{*} \\
(0,10)\end{array}$ & $\begin{array}{c}0,08^{* *} \\
(0,08)\end{array}$ & $\begin{array}{c}14^{* *} \\
(0,10)\end{array}$ & $\begin{array}{c}0,06^{* * *} \\
(0,04)\end{array}$ \\
\hline $\begin{array}{l}\text { Analyste en } \\
\text { renseignements } \\
\text { criminels }\end{array}$ & $\begin{array}{c}4,67 \\
(4,08)\end{array}$ & $\begin{array}{c}0,09^{*} \\
(0,09)\end{array}$ & $\begin{array}{c}0,06^{* *} \\
(0,06)\end{array}$ & $\begin{array}{c}0,46 \\
(0,35)\end{array}$ & $\begin{array}{c}0,19^{*} \\
(0,16)\end{array}$ \\
\hline Juge & $\begin{array}{c}1,51 \\
(0,99)\end{array}$ & $\begin{array}{c}0,16^{*} \\
(0,15)\end{array}$ & $\begin{array}{c}0,12^{*} \\
(0,10)\end{array}$ & $\begin{array}{c}0,37 \\
(0,27)\end{array}$ & $\begin{array}{c}0,15^{*} \\
(0,12)\end{array}$ \\
\hline Avocat & 3,02 & 0,22 & $0,16^{*}$ & 0,36 & $0,14^{* *}$ \\
& $(1,56)$ & $(0,18)$ & $(0,13)$ & $(0,22)$ & $(0,10)$ \\
\hline Psychiatre & 2,07 & - & 0,70 & 0,51 & 0,21 \\
& $(1,30)$ & $(0,71)$ & $(0,37)$ & $(0,17)$ \\
\hline Pédiatre & $\begin{array}{c}1,75 \\
(1,50)\end{array}$ & $\begin{array}{c}0,20 \\
(0,19)\end{array}$ & $\begin{array}{c}0,14 \\
(0,15)\end{array}$ & $\begin{array}{c}0,41 \\
(0,33)\end{array}$ & $\begin{array}{c}0,17 \\
(0,15)\end{array}$ \\
\hline Psychologue & $\begin{array}{c}11,50^{*} \\
(14,02)\end{array}$ & $\begin{array}{c}0,38 \\
(0,31)\end{array}$ & $\begin{array}{c}0,27 \\
(0,26)\end{array}$ & - & 0,41 \\
& $\begin{array}{c}3,47^{*} \\
(2,17)\end{array}$ & $\begin{array}{c}1,42 \\
(1,43)\end{array}$ & $\begin{array}{c}- \\
(0,34)\end{array}$ \\
\hline Travailleur social & $\begin{array}{c}1,02 \\
(0,02)\end{array}$ & $\begin{array}{c}0,99 \\
(0,01)\end{array}$ & $\begin{array}{c}0,99 \\
(0,01)\end{array}$ & $\begin{array}{c}1,00 \\
(0,01)\end{array}$ & $\begin{array}{c}1,00 \\
(0,01)\end{array}$ \\
\hline Âge & $\begin{array}{c}0,75 \\
(0,27)\end{array}$ & $\begin{array}{c}0,70 \\
(0,22)\end{array}$ & $\begin{array}{c}0,70 \\
(0,22)\end{array}$ & $\begin{array}{c}0,78 \\
(0,24)\end{array}$ & $\begin{array}{c}0,78 \\
(0,24)\end{array}$ \\
\hline Homme & $\begin{array}{c}0,80 \\
(0,44)\end{array}$ & $\begin{array}{c}0,62 \\
(0,31)\end{array}$ & $\begin{array}{c}0,62 \\
(0,31)\end{array}$ & $\begin{array}{c}1,17 \\
(0,46)\end{array}$ & $\begin{array}{c}1,17 \\
(0,46)\end{array}$ \\
\hline Connaissance du SJPA & $\begin{array}{c}0,95 \\
(0,40)\end{array}$ & $\begin{array}{c}0,70 \\
(0,30)\end{array}$ & $\begin{array}{c}0,70 \\
(0,30)\end{array}$ & $\begin{array}{c}1,64 \\
(0,68)\end{array}$ & $\begin{array}{c}1,64 \\
(0,68)\end{array}$ \\
\hline Canada & $\begin{array}{c}0,91 \\
(0,54)\end{array}$ & $\begin{array}{c}0,35 \\
(0,26)\end{array}$ & $\begin{array}{c}0,35 \\
(0,26)\end{array}$ & $\begin{array}{c}2,07 \\
(1,35)\end{array}$ & $\begin{array}{c}2,07 \\
(1,35)\end{array}$ \\
\hline
\end{tabular}

Note: Bien que nous ayons fait des régressions logistiques en utilisant systématiquement chaque spécialisation comme une catégorie de référence distincte, ce tableau présente seulement les résultats des analyses statistiquement significatives. Le Modèle $\mathbf{1}$ montre comment les policiers différaient des autres experts dans leurs opinions concernant l'impact des facteurs développementaux sur la responsabilité morale des jeunes (Effet élevé $=1 /$ Effet faible $=0$ ). Le Modèle 2 et le Modèle 3 montrent comment les psychiatres et les travailleurs sociaux, respectivement, diffèrent des autres experts dans leurs opinions concernant l'impact que les facteurs développementaux ont sur l'habileté des jeunes à subir un procès (Effet élevé = 1 / Effet faible $=0$ ). Le Modèles $\mathbf{4}$ et le $\mathbf{5}$ montrent comment les psychologues et les travailleurs sociaux, respectivement, diffèrent des autres spécialistes dans leurs opinions de la possibilité de réduire la culpabilité dans un cas particulier (Possibilité élevée $=1$ / Possibilité faible $=0$ ). ${ }^{*} p \leq 0,05 ;{ }^{* *} p \leq 0,01$; ${ }_{* \star *} \mathrm{p} \leq 0,001$; erreurs type entre parenthèses.

une grande influence aux facteurs développementaux dans l'aptitude des jeunes à subir un procès. Les travailleurs sociaux étaient $92 \%, 94 \%$, $88 \%$ et $84 \%$ plus susceptibles que, respectivement, les policiers $(\mathrm{p}<0,01)$, les analystes en renseignements criminels $(p<0,01)$, les juges $(p<0,05)$ 
FIG URE 2

La distribution des réponses à la question «Pensez-vous qu'il y ait des facteurs développementaux qui affectent l'aptitude des jeunes à subir un procès?" $(n=291)$

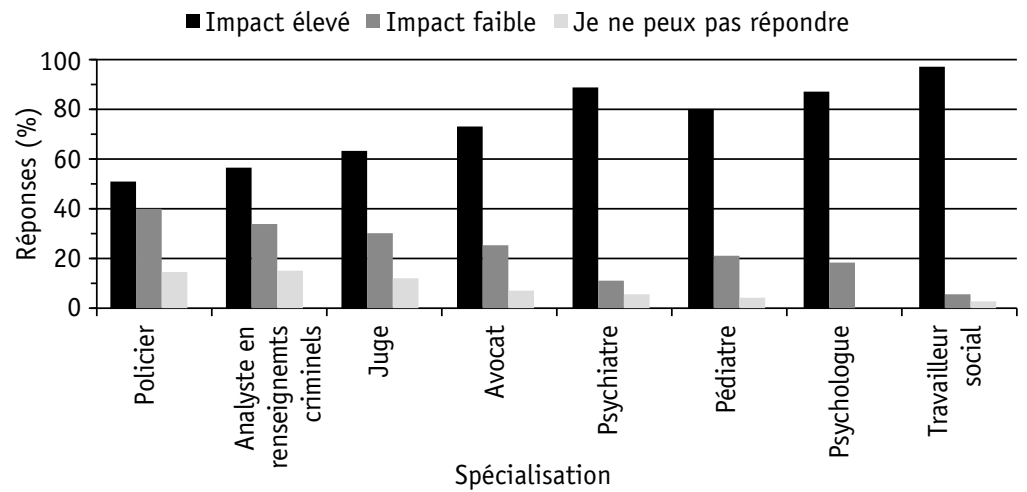

et les avocats ( $p<0,05)$, à affirmer que les facteurs développementaux influencent largement l'aptitude des jeunes à subir un procès.

\section{Détermination du degré de réduction de culpabilité}

Nous avons examiné les divergences entre les opinions des experts pour savoir jusqu'à quel point il leur était possible de réduire la culpabilité dans un cas particulier (voir la figure 3 pour une distribution des réponses par domaine d'expertise). Nous avons analysé nos données à l'aide de régressions logistiques multiples pour évaluer le lien entre l'expertise et la possibilité de déterminer le degré de réduction (élevé $=1$, faible $=0$ ) en utilisant chaque spécialisation comme une catégorie de référence. Selon nos analyses, les opinions des psychologues et des travailleurs sociaux différaient significativement de certains des autres groupes (voir tableau 2, modèles 4 et 5). Effectivement, les psychologues étaient $86 \%$ plus prédisposés que les policiers à indiquer qu'il est possible de déterminer le degré de réduction de la culpabilité dans un cas particulier $(\mathrm{p}<0,01)$. Les travailleurs sociaux étaient $94 \%, 81 \%$, $85 \%$ et $86 \%$ plus prédisposés que, respectivement, les policiers $(p<0,001)$, les analystes en renseignements criminels ( $p<0,05)$, les juges $(\mathrm{p}<0,05)$ et les avocats $(\mathrm{p}<0,01)$ à indiquer qu'il est tout à fait possible de déterminer la culpabilité individuelle d'un jeune $(\mathrm{p}<0,05)$. 
FIG URE 3

La distribution des réponses à la question «Est-il possible de déterminer le degré de réduction de la culpabilité dans un cas particulier de criminalité juvénile?" $(n=291)$

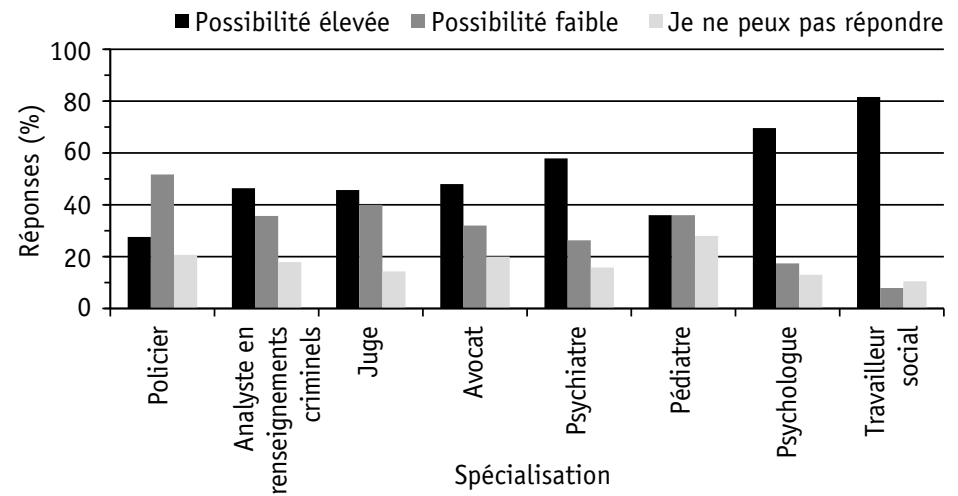

L'âge minimum de la juridiction juvénile

La figure 4 présente la distribution de fréquence des réponses en ce qui concerne les opinions quant à l'âge minimal pour déterminer la juridiction juvénile. Nous n'avons pas évalué ces données avec des régressions entre experts étant donné le manque de puissance statistique attribuable à cette question.

\section{F I G U RE 4}

La distribution des opinions sur l'âge minimal de juridiction juvénile

$$
(n=104, m=12,5 \text {, é.t. }=2,8)
$$

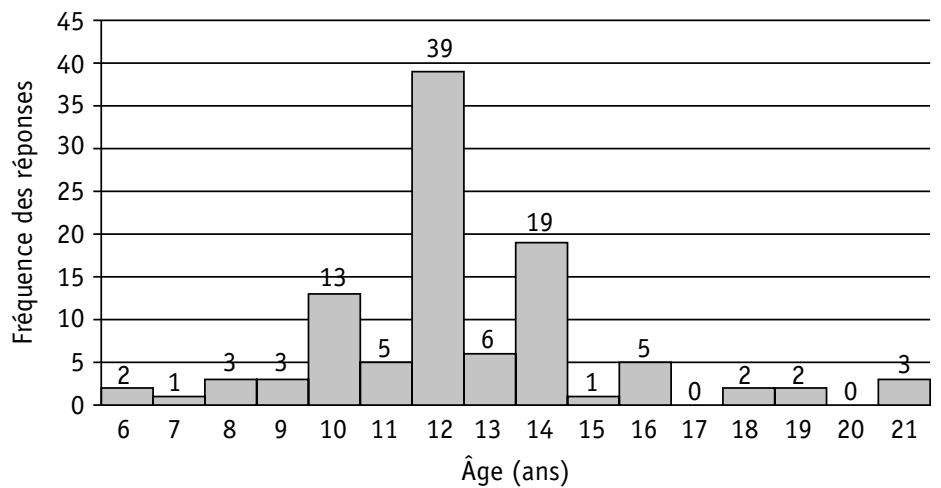




\section{Discussion}

L'ensemble de nos découvertes suggère que la communauté légale est raisonnablement consciente des effets des facteurs développementaux sur le comportement et les compétences légales des jeunes. Par exemple, la majorité des spécialistes légaux et cliniques s'entendent sur le fait que les facteurs développementaux ont un effet modéré à élevé sur la responsabilité morale des jeunes délinquants (figure 1) ainsi que sur leur aptitude à subir un procès (figure 2). Néanmoins, les policiers, comparés à d'autres experts, semblent négliger les déficits développementaux des jeunes délinquants. Plus précisément, les policiers étaient plus prédisposés que les experts cliniques à indiquer que les caractéristiques développementales des adolescents ont peu d'influence sur la responsabilité morale des jeunes ainsi que sur leur aptitude à subir un procès. Jusqu'à $38 \%$ des policiers ont indiqué que les déficits développementaux ont un rôle négligeable sur ces questions légales (se référer à la figure 1 et la figure 2). Ces résultats suggèrent qu'il est possible que le personnel policier interroge ou accuse des jeunes délinquants sans prendre leur jeune âge en compte. Effectivement, des recherches précédentes montrent que les policiers ont tendance à interroger les jeunes suspects comme ils interrogeraient des adultes (Feld, 2006; Meyer et Reppucci, 2007). De telles pratiques peuvent s'expliquer par le fait que les policiers ne reçoivent généralement pas de formation sur les effets potentiels négatifs des techniques interrogatoires, comme le risque accru de fausses confessions chez les jeunes (Drizin et Leo, 2004). Quelques recherches indiquent en effet que la formation peut jouer un rôle dans la sensibilité accrue de la police envers les déficits de développement des adolescents (Kostelnik et Reppucci, 2009).

Un manque de clémence des policiers envers les jeunes délinquants peut contribuer à la nature punitive des sentences juvéniles. En effet, en comparaison des autres experts légaux, les policiers sont ceux qui semblent posséder le plus de pouvoir discrétionnaire (Beckett, 1999). Le pouvoir d'arrestation, par exemple, est laissé à la discrétion du policier (Forst et Bushway, 2010). Des arrestations dans les cas où un avertissement pourrait être suffisant peuvent aggraver les peines des jeunes. En retour, le nombre de casiers judiciaires est un des facteurs qui détermine la sévérité des peines des jeunes puisqu'un nombre accru de casiers judiciaires entraîne des peines plus sévères (Roberts, 1997; Bushway et Piehl, 2007). De plus, la discrétion des juges dans la 
réduction des peines est souvent restreinte et, aux États-Unis, elle est limitée explicitement par le Code criminel. Bien que le système judiciaire du Canada accorde actuellement une certaine discrétion aux juges (Bala et al., 2009), un projet récent menace de réduire cette discrétion judiciaire (Loi sur la sécurité des rues et des communautés, 2011). Par conséquent, il est possible que les policiers détiennent plus de pouvoir sur les peines des jeunes que les juges.

Les adolescents peuvent être particulièrement susceptibles de faire preuve d'agressivité lorsqu'ils interagissent avec la police en raison des changements pubères (Kulin et al., 2000). Le comportement négatif d'un suspect, en retour, augmente les chances d'arrestation (Engel et al., 2000). Suivant cet ordre d'idées, des résultats de recherche suggèrent que les policiers sont plus susceptibles d'arrêter les jeunes que les adultes pour des délits similaires (Brown et al., 2009). Un meilleur encadrement de la discrétion policière pourrait aider à réduire le nombre de jeunes référés à la cour juvénile. D’ailleurs, la Loi sur le système de justice pénale pour les adolescents au Canada encourage les policiers à imposer des mesures extrajudiciaires aux jeunes (Carrington et Schulenberg, 2008). Par conséquent, nous suggérons qu'il est primordial de former le personnel de police pour interagir avec les jeunes délinquants en prenant en compte leurs déficits développementaux associés à l'adolescence afin de promouvoir un traitement juste des jeunes délinquants tout en minimisant le nombre de fausses condamnations.

Bien que la discrétion des juges puisse nuire à la conformité des peines (Doob et Cesaroni, 2004), il est important de considérer les caractéristiques personnelles des jeunes dans leur évaluation judiciaire puisqu'ils sont sujets à de larges différences individuelles dans leur maturité cognitive (Mayzera et al., 2009) en plus d'être sujets à être affectés par des maladies mentales (Soler, 2002). Afin d'individualiser le jugement des jeunes délinquants tout en conservant une conformité raisonnable entre les peines, la Loi canadienne sur le système de justice pénale pour les adolescents alloue une certaine discrétion aux juges tout en introduisant des consignes détaillées quant aux buts et aux principes qui doivent guider leur décision (Roberts et Bala, 2003). Malgré cet effort, les taux d'incarcération demeurent très variables à travers le Canada (Bala et al., 2009). Il est possible que le manque de conformité dans les peines des jeunes délinquants provienne de la difficulté qu'ont les juges à évaluer la responsabilité morale des jeunes dans des cas particuliers, ce qui influence en retour les décisions judiciaires. En effet, 
nos résultats suggèrent que $40 \%$ des juges croient qu'il est pratiquement, voire totalement impossible, de déterminer le degré de culpabilité individuelle (voir la figure 3). Il est donc essentiel de développer une approche structurée qui permettra d'évaluer la culpabilité juvénile tout en offrant les avantages du pouvoir discrétionnaire au sein d'une structure solide et cohérente dans l'attribution des peines. La science développementale pourrait contribuer à bâtir une partie de cette structure. Effectivement, une variété d'outils d'évaluation pourrait aider le système légal à jauger la maturité du jeune convoqué au tribunal. Ces outils mesurent des paramètres tels que l'impulsivité, le quotient intellectuel, l'habilité à résister à la pression des pairs et la perception des risques. Néanmoins, il peut être difficile d'utiliser ces paramètres de maturité comme des indicateurs directs de la responsabilité morale des jeunes. Une étroite collaboration entre les experts en science développementale et ceux de la justice juvénile pourrait cependant résoudre le problème et aider au perfectionnement des outils d'évaluation de la maturation afin d'augmenter leur validité auprès du système pénal.

Alors que les SJPA des États-Unis et du Canada adoptent depuis peu une approche plus punitive, on remarque que cette approche est accompagnée d'un déclin des politiques de réhabilitation (Cauffman et al., 1999; Snyder et Sickmund, 1999; Woolard et al., 2001; Redding et Frost, 2002; Andrews et Bonta, 2010). Ces nouvelles législations qui insistent sur une approche plus punitive envers les crimes des jeunes ont émergé en réponse à l'apparente préoccupation du public au sujet de la clémence du SJPA (Tufts et Roberts, 2002). Cependant, l'utilisation d'une approche punitive envers les jeunes délinquants va à l'encontre des propositions issues des recherches scientifiques qui soutiennent que les programmes de réhabilitation sont plus efficaces que la punition pour contrer les récidives (Forrest et al., 2000; Baerger et al., 2003; Day et al., 2004; Greenberg et al., 2007; Myers et Farrell, 2008; Vieira et al., 2009; Andrews et Bonta, 2010). De plus, la punition semble contribuer au développement des psychopathologies chez les jeunes, ce qui augmente les risques de récidive (Arredondo, 2003; Bonham, 2006; Cuellar et al., 2006; Fagan, 2008). Voilà pourquoi le système de justice semble largement inefficace en ce qui concerne la promotion de la sécurité publique.

Nous estimons qu'un dialogue plus serré entre science développementale et droit est nécessaire afin d'atteindre un consensus sur la manière appropriée de traiter les jeunes contrevenants tout en réduisant 
les taux de récidive criminelle. La science du développement peut, tout particulièrement, offrir ses savoirs au sujet du développement des adolescents au système de justice pénale pour les jeunes et ainsi contribuer à raffiner les politiques afin qu'elles aillent de pair avec les preuves scientifiques. Pour l'instant, l'adolescence, en tant qu'étape spécifique du développement, est très mal définie au sein du système légal alors que la définition de l'âge adulte dépend des politiques en vigueur. Par exemple, les adolescents acquièrent graduellement plus de responsabilités légales en atteignant des âges associés à différents privilèges, tels que le droit de conduire, le droit de voter, et le droit de consommer des boissons alcoolisées (Scott, 2000). De plus, les âges associés avec des responsabilités légales spécifiques varient selon les pays (Luciana, 2011). L'absence d'une définition claire de l'adolescence ainsi que l'ambiguïté présente entre adolescence et âge adulte peuvent contribuer à la difficulté qu'ont les experts juridiques à établir une tranche d'âge à laquelle la juridiction juvénile devrait toujours s'appliquer. Nos résultats indiquent que cette question demeure grandement controversée puisque les experts ne s'entendent pas sur l'âge minimal d'établissement d'une juridiction juvénile. Certains suggèrent que les jeunes devraient être tenus pénalement responsables dès 6 ans; d'autres suggèrent qu'ils devraient être tenus responsables seulement après 21 ans (figure 4). Une plus grande interaction entre science développementale et système légal pourrait permettre d'établir une étendue d'âges raisonnables en ce qui concerne une responsabilité pénale juvénile adaptée à leur étape développementale.

\section{Limites et mises en garde}

\section{Questionnaire}

Les résultats en science développementale influencent de plus en plus les décisions juridiques des jeunes (Roper c. Simmons, 2005 ; R. c. D. B., 2008; Graham c. Florida, 2010). Comprendre la position des experts légaux par rapport à la recherche développementale est donc primordial afin d'étudier l'influence de ces découvertes sur le SJPA. L'opinion généralement favorable au sujet de la science développementale chez les spécialistes légaux suggère qu'un dialogue plus serré entre le système pénal et la science du développement est possible. Une telle collaboration pourrait permettre au système légal de déterminer la culpabilité des adolescents tout en promouvant leur développement. 
Aucune étude n'a, à notre connaissance, exploré les opinions des experts légaux et cliniques sur les implications des connaissances nouvelles apportées par les recherches en science développementale en ce qui concerne la responsabilité morale des jeunes dans le contexte de la justice pénale.

Notre sondage constitue un projet pilote visant à obtenir un aperçu de l'opinion générale des différents experts en ce qui a trait à la responsabilité morale des jeunes dans le contexte de la justice pénale. Par conséquent, les questions du sondage étaient peu spécifiques. Par exemple, puisque l'âge de la responsabilité criminelle demeure une question controversée (Urbas, 2000), nous avons dû omettre de spécifier la tranche d'âge à laquelle nos questions se référaient. Cependant, ce manque de précision était aussi un avantage puisqu'il nous permettait de désigner les adolescents par le simple terme «jeunes», omettant ainsi les stéréotypes liés à l'âge et permettant une approximation plus représentative de l'opinion des experts envers le rôle des facteurs développementaux, lesquels dépendent plus de la maturité que de l'âge. De plus, l'utilisation du terme imprécis «jeunes» pour désigner les adolescents nous a permis de percevoir le rôle que les spécialistes attribuent aux facteurs développementaux sans pour autant introduire de biais associé avec les stéréotypes d'une tranche d'âge précise. Une telle conceptualisation fournit des réponses plus riches puisque les caractéristiques développementales sont plus fortement reliées à la maturité que ne l'est l'âge. Malgré cela, il y a lieu d'admettre que la formulation des questions a pu affecter les réponses et que les réponses à des questions vagues peuvent être particulièrement difficiles à interpréter (Steinberg et Piquero, 2010). Ci-dessous, nous énumérons les divers problèmes d'interprétation qui peuvent émaner de la formulation de nos questions.

Tout d'abord, la formulation de nos questions présuppose que les adolescents présentent une culpabilité moins importante que celle des adultes. Cette prémisse provient des systèmes de justice pénale des pays dans lesquels nous avons distribué notre sondage; chacun de ces pays a un système de justice juvénile plus tolérant que celui des adultes. Ainsi, notre questionnaire ne contenait aucune question comparant la responsabilité morale des jeunes à celle des adultes et supposait plutôt que les jeunes étaient considérés comme moins coupables que les adultes. Nous avons plutôt cherché à évaluer l'importance attribuée par les experts aux facteurs développementaux dans la détermination de leur culpabi- 
lité réduite. Ainsi, nos résultats illustrent certaines tendances générales chez les experts légaux et cliniques dans leur perception des implications du savoir issu de la science développementale sur le système de justice des jeunes.

Deuxièmement, il est possible que l'interprétation du concept «d'aptitude à subir un procès» ait pu différer, selon les experts, vu le peu de reconnaissance attribuée dans le passé aux déficits développementaux comme facteur de réduction de cette aptitude (Bonnie et Grisso, 2000; Redding et Frost, 2002), ainsi qu'à l'association fréquente entre ce concept et la psychopathologie (Grisso, 2005). De ce fait, il est possible que certains experts n'aient pas pris l'immaturité développementale en compte en ce qui a trait à l'aptitude à subir un procès. Il est également possible que les experts légaux aient eu tendance à comparer l'aptitude des jeunes à subir un procès avec celle des individus atteints de psychopathologies. Cette dernière étant particulièrement basse, un tel point de comparaison pourrait expliquer le faible rôle des facteurs développementaux dans l'aptitude des jeunes à subir un procès telle qu'estimée par les experts légaux. Nous sommes donc conscients que nos résultats ne permettent pas de discerner si les experts légaux ont une connaissance moindre de l'immaturité des jeunes quant à leur aptitude à subir un procès ou s'ils ont simplement un point de repère différent car ils sont habitués à prendre en compte une faible aptitude chez les individus atteints de problèmes psychopathologiques. Malgré cela, nos découvertes indiquent qu'une grande proportion des experts considèrent que l'immaturité développementale joue un rôle important dans l'aptitude des jeunes à subir un procès. Si cette question n'était pas influencée par cette apparente ambiguiité, les experts légaux qui affirment que les traits développementaux affectent négativement l'aptitude des jeunes à subir un procès auraient été plus nombreux que ce que nos résultats ne laissent paraître.

Finalement, il est possible que les opinions des experts sur la responsabilité morale des jeunes changent selon l'information à laquelle ils ont accès concernant l'accusé: son âge, le crime commis et la peine imposée (Steinberg et Piquero, 2010). Par conséquent, nous faisons preuve d'un enthousiasme limité en ce qui a trait à l'utilisation de ces résultats de recherche dans l'établissement de nouvelles politiques concernant la responsabilité morale des jeunes au sein des systèmes de justice juvénile. Ces résultats devront aussi servir à la formulation de nouvelles questions de recherche dans lesquelles des objectifs de recher- 
che plus spécifiques nous permettront de mieux cerner le rôle joué par l'âge de l'accusé et le type de crime commis sur les opinions des experts a sujet de la responsabilité morale des jeunes.

\section{Le sondage en ligne}

L'utilisation d'un sondage en ligne apporte divers avantages et inconvénients. Afin d'illustrer ces caractéristiques, nous référons le lecteur à un numéro spécial de la revue Public Opinion Quarterly (vol. 72, $\mathrm{n}^{\circ}$ 5, 2008). Les taux de réponse des sondages en ligne utilisant des invitations par courriel dépassent ceux d'autres médias électroniques, tels que les messages textes sur des appareils téléphoniques, sans pour autant compromettre la composition de l'échantillon des répondants (Bosnjak et al., 2007). Les taux de réponse pour des sondages en ligne semblables à celui utilisé dans la présente étude diffèrent de ceux des sondages postaux (Matz, 1999; Dillman, 2000; Underwood et al., 2000; Manfreda et al., 2008) présentant un taux de réponse de typiquement moins de 10\% (Crawford, Couper et Lamias, 2001 ; Smith, 1997; Tse et al., 1995 ; Witmer, Colman et Katzman, 1999). Bien qu'il soit difficile d'estimer le taux de réponse à notre sondage, le projet pilote s'est allié avec de récents sondages (Sherman et Hickner, 2008) et soutenait l'option d'entreprendre un sondage en ligne (Callegaro et Disogra, 2008; Couper et Miller, 2008; Kreuter et al., 2008) dans lequel la représentativité des réponses compenserait largement un éventuel faible taux de participation (Cook et al., 2000).

Les sondages par Internet ont l'avantage de permettre une collection de données efficace et rapide (Couper et Miller, 2008). Ils offrent la possibilité d'avoir un grand échantillon tout en réduisant les coûts qui y sont normalement reliés (Dillman, 2000) et ne requièrent pas d'adresse complète, procurant ainsi une garantie d'anonymat aux répondants (Eysenbach et Wyatt, 2002). Conséquemment, les répondants des sondages en ligne ont généralement plus d'aisance à répondre à des questions controversées et sont moins affectés par les jugements sociaux dans leurs réponses (Couper et al., 2001 ; Pealer et al., 2001 ; Kreuter et al., 2008). Cet atout est un grand avantage lorsqu'il s'agit de sujets sensibles et de politiques, tel que l'est la responsabilité morale de la jeunesse.

Malgré cet avantage considérable, les sondages en ligne ont le désavantage d'exclure les réponses d'individus n'ayant pas accès à Internet, 
ce qui introduit un biais dans l'échantillonnage couvert (Couper, 2000, 2007). Cependant, la majorité des experts visés par notre sondage ont accès à Internet, ce qui minimise l'erreur de couverture de la présente enquête (Dillman, 2000; Martin, 2003). Un autre désavantage des sondages par Internet est la possibilité que certains répondent au sondage à plusieurs reprises. Afin d'éviter des réponses dédoublées, nous avons mis en place des mesures restrictives, comme l'utilisation de cookies et la surveillance d'adresses IP (Couper, 2007). Un dernier désavantage que nous tenons à souligner est la possibilité de divergence entre les répondants et les non-répondants en termes de démographie et d'attitudes, communément appelé le biais de non-réponse (Umbach, 2004). Néanmoins, de récentes études sur les sondages en ligne démontrent que les différences démographiques entre les répondants et les non-répondants sont minimes et comparables à celles des sondages postaux (Sax et al., 2008). De plus, la littérature sur les sondages en ligne ne contient aucune mention d'un biais basé sur les caractéristiques démographiques.

Dans le présent sondage, nous utilisons un échantillon auto-sélectionné afin d'évaluer les opinions des spécialistes cliniques et légaux au sujet des procédures pénales des jeunes. Il est théoriquement possible que les experts ayant choisi de compléter notre sondage soient a priori plus enclins à avoir une opinion extrême sur le sujet. De ce fait, il est possible que nos résultats soient biaisés, estimant disproportionnellement l'opinion des spécialistes les plus conscients des implications en science développementale en ce qui concerne la responsabilité morale des jeunes et leur aptitude à subir un procès. Plus précisément, puisque le débat à propos de la responsabilité pénale des adolescents acquiert de plus en plus d'attention (Baerger et al., 2003; Maroney, 2009), il est probable que les partisans d'une réforme du SJPA soient plus aptes à exprimer leurs opinions que les experts en défaveur de la réforme. Effectivement, l'exploration de nos données suggère que la distribution des réponses de tous les spécialistes tend en faveur de l'acceptation du rôle des facteurs développementaux comme facteur décisionnel dans la responsabilité morale des jeunes. De ce fait, dans l'éventualité d'un biais d'échantillonnage, le fossé entre la communauté légale et la science du développement pourrait être plus grand que ce que nos découvertes laissent entendre. 


\section{Conclusion}

Nos découvertes suggèrent que la communauté légale est modérément sensible à la recherche développementale reliée à la responsabilité morale des jeunes. Malgré cela, les policiers semblent être enclins à négliger les déficits développementaux des jeunes délinquants. Puisqu'ils sont en mesure d'avoir un effet sur les peines des jeunes en raison du pouvoir discrétionnaire qu'ils exercent lors des arrestations, il est important qu'ils reçoivent une formation sur les déficits développementaux des jeunes ainsi que sur la manière appropriée d'interagir avec eux. Une telle approche pourrait réduire le nombre d'arrestations injustifiées et de fausses confessions. Il semble en effet que le système légal tirerait avantage d'une interaction plus grande avec le savoir issu des sciences du développement. Les variations individuelles dans le développement des jeunes sont ainsi des connaissances qui permettraient à des officiers légaux mieux formés d'exercer plus latitude discrétionnaire dans leurs décisions. Ces quelques suggestions contribueraient grandement à améliorer la mise en œuvre des connaissances nouvelles au sujet du développement des adolescents en ce que concerne leur responsabilité morale et contribueraient au débat concernant l'amélioration du système de justice des jeunes.

\section{Références}

Agnew, R. (2003). An integrated theory of the adolescent peak in offending. Youth and Society, 34(3), 263-299.

Andrews, D. A., \& Bonta, J. (2010). Rehabilitating criminal justice policy and practice. Psychology, Public Policy and Law, 16, 39-85.

Arredondo, D. E. (2003). Child development, children's mental health and the juvenile justice system. Stanford Law and Policy Review, 14, 13-28.

Baerger, D. R., Griffin, E. F., Lyons, J. S., \& Simmons, R. (2003). Competency to stand trial in preadjudicated and petitioned juvenile defendants. Journal of the American Academy of Psychiatry and the Law Online, 31(3), 314-320.

Bala, N., Carrington, P., \& Roberts, J. (2009). Evaluating the Youth Criminal Justice Act after five years: A qualified success. Canadian Journal of Criminology and Criminal Justice/La Revue Canadienne de Criminologie et de Justice Pénale, 51(2), 131-167.

Beckett, K. (1999). Making crime pay: Law and order in contemporary American politics: Oxford University Press, USA.

Blakemore, S. J., \& Choudhury, S. (2006). Development of the adolescent brain: Implications for executive function and social cognition. Journal of Child Psychology and Psychiatry, 47(3-4), 296-312. 
Bonham, E. (2006). Adolescent mental health and the juvenile justice system. Pediatric Nursing, 32(6), 591-595.

Bonnie, R. J., \& Grisso, T. (2000). Adjudicative competence and youthful offenders. In T. Grisso \& R. G. Schwartz (eds.), Youth on trial: A developmental perspective on juvenile justice (73-103). Chicago: University of Chicago Press.

Bosnjak, M., Neubarth, W., Couper, M. P., Bandilla, W., \& Kaczmirek, L. (2007). Prenotification in web-based access panel surveys: The influence of mobile text messaging versus e-mail on response rates and sample composition. Social Science Computer Review, 0894439307305895. doi: $10.1177 / 0894439307305895$

Brown, R. A., Novak, K. J., \& Frank, J. (2009). Identifying variation in police officer behavior between juveniles and adults. Journal of Criminal Justice, 37(2), 200-208.

Burleson, B. R. (2003). The experience and effects of emotional support: What the study of cultural and gender differences can tell us about close relationships, emotion, and interpersonal communication. Personal Relationships, 10(1), 1-23.

Bushway, S. D., \& Piehl, A. M. (2007). The inextricable link between age and criminal history in sentencing. Crime and Delinquency, 53(1), 156-183.

Callegaro, M., \& Disogra, C. (2008). Computing response metrics for online panels. Public Opinion Quarterly, 72(5), 1008-1032.

Carrington, P. J., \& Schulenberg, J. L. (2008). Structuring police discretion. Criminal Justice Policy Review, 19(3), 349-367.

Cauffman, E., \& Steinberg, L. (2000a). (Im)maturity in adolescence: Why adolescents may be less culpable than adults. Behavioral Sciences and the Law, $18,741-760$.

Cauffman, E., \& Steinberg, L. (2000b). Researching adolescents' judgment and culpability. In T. Grisso \& R. G. Schwartz (eds.), Youth on trial: A developmental perspective on juvenile justice (325-343). Chicago: University of Chicago Press.

Cauffman, E., Woolard, J., \& Reppucci, N. D. (1999). Justice for juveniles : New perspectives on adolescents' competence and culpability. Quinnipiac Law Review, 18, 403-913.

Cesaroni, C., \& Bala, N. (2008). Deterrence as a principle of youth sentencing: No effect on youth, but a significant on judges. Queen's Law Journal, 34, 447.

Cook, C., Heath, F., \& Thompson, R. L. (2000). A meta-analysis of response rates in Web-or Internet-based surveys. Educational and Psychological Measurement, 60(6), 821-836.

Couper, M. P. (2000). Review: Web surveys: A review of issues and approaches. Public Opinion Quarterly, 64(4), 464-494.

Couper, M. P. (2007). Issues of representation in eHealth research (with a focus on web surveys). American Journal of Preventive Medicine, 32(5), S83-S89.

Couper, M. P., \& Miller, P. V. (2008). Web survey methods: Introduction. Public Opinion Quarterly, 72(5), 831-835. 
Couper, M. P., Tourangeau, R., \& Steiger, D. M. (2001). Social presence in web surveys. Paper presented at the SIGCHI Conference on Human Factors in Computing Systems, Seattle, Washington, United States.

Crawford, S. D., Couper, M. P., \& Lamias, M. J. (2001). Web surveys : Perceptions of burden. Social Science Computer Review, 19(2), 146-162.

Cuellar, A. E., McReynolds, L. S., \& Wasserman, G. A. (2006). A cure for crime: Can mental health treatment diversion reduce crime among youth? Journal of Policy Analysis and Management, 25(1), 197-214.

Day, A., Howells, K., \& Rickwood, D. (2004). Current trends in the rehabilitation of juvenile offenders. Trends and Issues in Crime and Criminal Justice.

Diamond, A. (2006). The early development of executive functions. Lifespan Cognition: Mechanisms of Change, 70-95.

Dillman, D. A. (2000). Mail and Internet surveys: The Tailored design method (2nd edition ed.). New York: John Wiley and Sons.

Drizin, S. A., \& Leo, R. A. (2004). The problem of false confessions in the post-DNA world. North Carolina Law Review, 82, 891-2155.

Engel, R. S., Sobol, J. J., \& Worden, R. E. (2000). Further exploration of the demeanor hypothesis: The interaction effects of suspects' characteristics and demeanor on police behavior. Justice Quarterly, 17(2), 235-258.

Eysenbach, G., \& Wyatt, J. (2002). Using the Internet for surveys and health research. Journal of Medical Internet Research, 4(2), e13.

Fagan, J. (2008). Juvenile crime and criminal justice: Resolving border disputes. The Future of Children, 18(2), 81-118.

Feld, B. C. (2006). Police interrogation of juveniles: An empirical study of policy and practice. The Journal of Criminal Law and Criminology, 219-316.

Forrest, C. B., Tambor, E., Riley, A. W., Ensminger, M. E., \& Starfield, B. (2000). The health profile of incarcerated male youths. Pediatrics, 105(1), 286-291.

Forst, B., \& Bushway, S. D. (2010). Discretion, rule of law, and rationality. Paper presented at the The Past and Future of Empirical Sentencing Research, Albany, New York.

Galligan, D. J. (1990). Discretionary powers: A legal study of official discretion. Oxford: Clarendon Press.

Gardner, W., \& Steinberg, L. (2005). Peer influence on risk taking, risk preference, and risky decision making in adolescence and adulthood: An experimental study. Developmental Psychology, 41, 625-635.

Giedd, J. N., Blumenthal, J., Jeffries, N. O., Castellanos, F. X., Liu, H., Zijdenbos, A., Paus, T. Evans, A. C., \& Rapoport, J. L. (1999). Brain development during childhood and adolescence: A longitudinal MRI study. Nature Neuroscience, 2, 861-862.

Gottfredson, M. R., \& Hirschi, T. (1990). A general theory of crime. Stanford: Stanford University Press.

Greenberg, M. T., Riggs, N. R., \& Blair, C. (2007). The role of preventive interventions in enhancing neurocognitive functioning and promoting competence in adolescence. Adolescent Psychopathology and the Developing Brain: Integrating Brain and Prevention Science, 441-461. 
Greenberger, E., \& Sørensen, A. B. (1974). Toward a concept of psychosocial maturity. Journal of Youth and Adolescence, 3(4), 329-358.

Grisso, T. (2005). Clinical evaluations for juveniles' competence to stand trial: A guide for legal professionals. Sarasota: Professional Resource Press.

Jackson, R. (2008). Learning forensic assessment. New York: Routledge.

Jennings, J. C. (2010). Juvenile Justice, Sullivan, and Graham: How the Supreme Court's decision will change the neuroscience debate. Duke Law and Technology Review, 6-7.

Kambam, P., \& Thompson, C. (2009). The development of decision-making capacities in children and adolescents: Psychological and neurological perspectives and their implications for juvenile defendants. Behavioral Sciences and the Law, 27(2), 173-190.

Keating, D. P. (2004). Cognitive and brain development. Handbook of adolescent psychology, 2, 45-84.

Klauer, T., \& Winkeler, M. (2002). Gender, mental health status, and social support during a stressful event. Heart disease: Environment, stress, and gender, 327, 223-236.

Kostelnik, J. O., \& Reppucci, N. D. (2009). Reid training and sensitivity to developmental maturity in interrogation: Results from a national survey of police. Behavioral Sciences and the Law, 27, 361-379.

Kreuter, F., Presser, S., \& Tourangeau, R. (2008). Social desirability bias in CATI, IVR, and web surveys. Public Opinion Quarterly, 72(5), 847-865.

Kulin, H. E., Finkelstein, J. W., Susman, E., Chinchilli, V., Liben, L., Schwab, J., Demers, L., \& D'Arcangelo, M. R. (2000). The role of sex hormones on the control of behavior during human puberty. In J. P. Bourguignon \& $\mathrm{T}$. M. Plant (Eds.), Onset of Puberty in Perspective (Vol. 1202, pp. 389-405). Amsterdam: Elsevier Science Bv.

Laberge, D., Morin, D., \& Robert, M. (1995). La réforme du Code criminel canadien en matière de troubles mentaux et son impact sur la détention des justiciables. Criminologie, 28(2), 61-83.

Luciana, M. (2011). Development of the adolescent brain: Neuroethical implications for the understanding of executive function and social cognition. In J. Illes \& B. J. Sahakian (Eds.), The Oxford handbook of neuroethics. New York: Oxford University Press.

Luna, B., Bunge, S., Schlaggar, B. L., Casey, B. J., Klingberg, T., Vaidya, C.,... Tapert, S. F. (2009, July 16).

Magar, E. C. E., Phillips, L. H., Hosie, \& A, J. (2008). Self-regulation and risk-taking. Personality and Individual Differences, 45(2), 153-159.

Manfreda, K. L., Bosnjak, M., Berzelak, J., Haas, I., \& Vehovar, V. (2008). Web surveys versus other survey modes: A meta-analysis comparing response rates. International Journal of Market Research, 50(1), 79-104.

Maroney, T. A. (2009). The false promise of adolescent brain science in juvenile justice. Notre Dame Law Review, 85(1), 89-176.

Martin, S. (2003). MDs' office Internet use hits 57\%. Canadian Medical Association Journal, 168(4), 475. 
Matz, C. M. (1999). Administration of web versus paper surveys: Mode effects and response rates. (Master of Science), University of North Carolina, Chapel Hill.

Maxwell, K. A. (2002). Friends: The role of peer influence across adolescent risk behaviors. Journal of Youth and Adolescence, 31(4), 267-277.

Meyer, J. R., \& Reppucci, N. D. (2007). Police practices and perceptions regarding juvenile interrogation and interrogative suggestibility. Behavioral Sciences and the Law, 25(6), 757-780.

Myers, D. M., \& Farrell, A. F. (2008). Reclaiming lost opportunities: Applying public health models in juvenile justice. Children and Youth Services Review, 30(10), 1159-1177.

Pealer, L. N., Weiler, R. M., Pigg, R. M., Miller, D., \& Dorman, S. M. (2001). The feasibility of a web-based surveillance system to collect health risk behavior data from college students. Health Education and Bebavior, 28(5), 547-559.

Pfaff, J. F. (2006). The continued vitality of structured sentencing following Blakely: The effectiveness of voluntary guidelines. UCLA Law Review, 54, 235-308.

Raz, A., \& Buhle, J. (2006). Typologies of attentional networks. Nature Reviews Neuroscience, 7(5), 367-379.

Redding, R. E., \& Frost, L. E. (2002). Adjudicative competence in the modern juvenile court. Virginia Journal of Social Psychology and Law, 9, 353-410.

Roberts, J. V. (1997). The role of criminal record in the sentencing process. Crime and Justice, 22, 303-362.

Santor, D. A., Messervey, D., \& Kusumakar, V. (2000). Measuring peer pressure, popularity, and conformity in adolescent boys and girls: Predicting school performance, sexual attitudes, and substance abuse. Journal of Youth and Adolescence, 29(2), 163-182.

Sax, L., Gilmartin, S., Lee, J., \& Hagedorn, L. S. (2008). Using web surveys to reach community college students: An analysis of response rates and response bias. Community College Journal of Research and Practice, 32(9), 712-729.

Scott, E. S. (2000). The legal construction of adolescence. Hofstra Law Review, 29, 547-598.

Scott, E. S., \& Grisso, T. (2004). Developmental incompetence, due process, and juvenile justice policy. Public Law and Legal Theory Working Paper Serie, $1-11$.

Scott, E. S., \& Steinberg, L. (2008). Retbinking juvenile justice. Cambridge: Harvard University Press.

Sherman, R., \& Hickner, J. (2008). Academic physicians use placebos in clinical practice and believe in the mind-body connection. Journal of General Internal Medicine, 23(1), 7-10.

Shonkoff, J. P., \& Phillips, D. (2000). From neurons to neighborboods: The science of early childhood development. Washington: National Academy Press.

Shook, J. J., Vaughn, M. G., Litschge, C., Kolivoski, K., \& Schelbe, L. (2009). The importance of friends among foster youth aging out of care: Cluster profiles of deviant peer affiliations. Children and Youth Services Review, 31(2), 284-291. 
Smith, C. B. (1997). Casting the net: Surveying an Internet population. Journal of Computer-Mediated Communication, 3(1).

Snyder, H. N., \& Sickmund, M. (1999). Juvenile offenders and victims: 1999 National Report. Washington: Office of Juvenile Justice and Delinquency Prevention.

Sowell, E. R., Thompson, P. M., Holmes, C. J., Jernigan, T. L., \& Toga, A. W. (1999). In vivo evidence for postadolescent brain maturation in frontal and striatal regions. Nature Neuroscience, 2, 859-860.

Spear, L. P. (2000). The adolescent brain and age-related behavioral manifestations. Neuroscience and Biobehavioral Reviews, 24(4), 417-463.

Steinberg, L., \& Piquero, A. R. (2010). Manipulating public opinion about trying juveniles as adults: An experimental study. Crime $\mathcal{\sigma}$ Delinquency, 56(4), 487-506.

Steinberg, L., \& Scott, E. S. (2003). Less guilty by reason of adolescence: Developmental immaturity, diminished responsibility, and the juvenile death penalty. American Psychologist, 58, 1009-1018.

Tse, A. C. B., Tse, K. C., Yin, C. H., Ting, C. B., Yi, K. W., Yee, K. P., \& Hong, W. C. (1995). Comparing two methods of sending out questionnaires: E-mail versus mail. Journal of the Market Research Society, 37(4), 441-446.

Tufts, J., \& Roberts, J. V. (2002). Sentencing juvenile offenders: Comparing public preferences and judicial practice. Criminal Justice Policy Review, 13(1), 46-64.

Umbach, P. D. (2004). Web surveys: Best practices. New Directions for Institutional Research, 2004(121), 23-38.

Underwood, D., Kim, H., \& Matier, M. (2000). To mail or to web: Comparisons of survey response rates and respondent characteristics. Paper presented at the Annual Forum of the Association for Institutional Research, Cincinnati, Ohio.

Urbas, G. (2000). The age of criminal responsibility. Trends and Issues in Crime and Criminal Justice, 181, 1-6.

Vieira, T. A., Skilling, T. A., \& Peterson-Badali, M. (2009). Matching courtordered services with treatment needs. Criminal Justice and Behavior, 36(4), 385-401.

Viljoen, J. L., \& Grisso, T. (2007). Prospects for remediating juveniles' adjudicative incompetence Psychology, Public Policy and Law, 13, 87-273.

Viljoen, J. L., \& Roesch, R. (2005). Competence to waive interrogation rights and adjudicative competence in adolescent defendants: Cognitive development, attorney contact, and psychological symptoms. Law and Human Bebavior, 29(6), 723-742.

White, H. (1980). A heteroskedasticity-consistent covariance matrix estimator and a direct test for heteroskedasticity. Econometrica, 48(4), 817-838.

Witmer, D. F., Colman, R. W., \& Katzman, S. L. (1999). From paper-and-pencil to screen-and-keyboard: Toward a methodology for survey research on the Internet. In S. Jones (Ed.), Doing Internet research : Critical issues and methods for examining the Net (pp. 145-161). 
Woolard, J. L., Fondacaro, M. R., \& Slobogin, C. (2001). Informing juvenile justice policy: Directions for behavioral science research. Law and Human Behavior, 25(1), 13-24.

Yurgelun-Todd, D. (2007). Emotional and cognitive changes during adolescence. Current Opinion in Neurobiology, 17(2), 251-257.

\section{Les lois}

\section{Case Law}

Graham c. Florida. (2010). 78 U.S.L.W. 4387.

R. c. D.B. (2008). S.C.C. 25.

Roper c. Simmons. (2005). 543 U.S. 551.

\section{Loi canadienne}

Loi sur la sécurité des rues et des communautés. Ministère de la justice, Canada.

ABSTRACT - Evidence from developmental science sheds new light on legal aspects pertaining to the blameworthiness of youth; however, incorporating these findings into the youth criminal justice system has been largely unsuccessful. Using an online survey, we probed the opinions, beliefs, and attitudes of legal and clinical specialists concerning the extent to which developmental parameters affect youth culpability and their ability to stand trial. Our findings suggest that while the majority of legal and clinical experts acknowledge the impact of developmental factors on the legal responsibility of youth, others (e.g., law enforcement) often underestimate such data. Here we outline how a closer dialogue between law professionals and developmental scientists will better facilitate a sorely overdue legal practice that fashions itself more closely after evidence-based science.

KEYWORDS - Brain development, adolescence, blameworthiness, adjudicative competence, youth criminal justice system.

RESUMEN - Recientemente, las investigaciones sobre el desarrollo cognitivo son parte de una gran fuente de información referente a la responsabilidad moral atribuible a los delincuentes juveniles. Sin embargo, la integración de dichos conocimientos en el sistema de justicia de menores se encuentra muy limitada. Utilizando el sistema informático gratuito en línea, LimeSurvey, hemos evaluado las opiniones, las creencias y las actitudes de los expertos legales (Ej. Los oficiales de policía, los abogados, los jueces) y clínicos (Ej. los psicólogos, los psiquiatras, los trabajadores sociales) en lo referente a el impacto de los factores de desarrollo sobre el nivel de responsabilidad de los jóvenes y de su aptitud a someterse a un proceso judicial. Nuestros resultados sugieren que, mientras la mayoría de los expertos legales y clínicos reconocen el rol de la ciencia del desarrollo en la justicia penal, algunos expertos (Ej. los oficiales de policía) subestiman su importancia. Nosotros sostenemos que un diálogo mas cercano entre la ciencia del desarrollo y el sistema de justicia es necesario para conciliar las prácticas legales con los descubrimientos científicos actuales.

PALABRAS CLAVES - Desarrollo cerebral, adolescencia, culpabilidad, aptitud para ser juzgado, sistema de justicia penal de menores. 


\section{A N NEXE}

\section{Sondage}

1. Veuillez indiquer votre sexe:

- Masculin

- Féminin

2. Veuillez indiquer votre âge:

3. Dans quel pays êtes-vous situé?

4. Veuillez identifier votre groupe d'appartenance

Chercheur

Veuillez préciser:

- science développementale

- criminalistique

- science cognitive

- science sociale

- neuroscience

- criminologie

- Autre:

Médecin

Veuillez préciser :

- psychiatrie générale

- psychiatrie des jeunes et des adolescents

- pédiatrie

- Autre:

Professionnel de la santé

Veuillez préciser:

- Travailleur social

- Psychologue

- Autre:

Professionnel de la loi

Veuillez préciser:

- Juriste

- Avocat

- Juge

- Autre: 
Renforcement juridique:

Veuillez préciser:

- Détective

- Policier

- Agent de probation

- Autre:

5. Veuillez évaluer votre niveau de connaissance du système de justice juvénile ( 1 = aucune connaissance $/ 5=$ extrêmement connaissant)

6. Veuillez évaluer les facteurs suivants selon leur influence potentielle envers le comportement délinquant des adolescents: $(1=$ aucun effet $/ 5=$ effet important / Je ne peux pas répondre):

- Les parties frontales du cerveau sous-développées

- Un haut niveau de testostérone (chez les garçons)

- L'immaturité psycho-sociale

- Une réduction de l'aversion au risque

- Une grande susceptibilité à la pression des pairs

- Une réduction de l'aptitude à évaluer les conséquences de ses actes

- Une réduction du contrôle de l'inhibition

- Une réduction du contrôle de soi

- L'humeur instable

- Une perte du contrôle cognitif et émotionnel de soi

- Une vulnérabilité au stress accrue

- Difficulté dans la prise de décision

- Le manque d'expérience

- La formation de son identité poussant à expérimenter

- L'environnement (les parents, le statut socioéconomique)

7. Pensez-vous qu'il y ait d'autres facteurs développementaux qui affectent le comportement délinquant des adolescents?

- Oui

- Veuillez spécifier le ou les facteurs et indiquer leur importance entre parenthèses: $(1=$ aucun effet $/ 5=$ effet important $)$

- Non

- Je ne peux pas répondre 
8. Pensez-vous qu'il y ait des traits développementaux ayant une influence sur le niveau de responsabilité des jeunes? $(1=$ non $/ 5$ = certainement / Je ne peux pas répondre)

Veuillez identifier les traits développementaux qui, selon vous, déterminent la responsabilité morale réduite des jeunes:

- Les parties frontales du cerveau sous-développées

- Un haut niveau de testostérone (chez les garçons)

- L'immaturité psycho-sociale

- Une réduction de l'aversion au risque

- Une grande susceptibilité à la pression des pairs

- Une réduction de l'aptitude à évaluer les conséquences de ses actes

- Une réduction du contrôle de l'inhibition

- Une réduction du contrôle de soi

- L'humeur instable

- Une perte du contrôle cognitif et émotionnel de soi

- Une vulnérabilité au stress accrue

- Difficulté dans la prise de décision

- Le manque d'expérience

- La formation de son identité poussant à expérimenter

- L'environnement (les parents, le statut socioéconomique)

- Autre:

9. Est-il possible de déterminer le degré de réduction de la responsabilité morale dans un cas particulier de criminalité juvénile? $(1=$ impossible $/ 5=$ très possible / Je ne peux pas répondre)

9a. Veuillez préciser la manière avec laquelle il serait possible de déterminer le degré de réduction de responsabilité morale d'un jeune:

- Selon son âge

- Selon son Q.I.

- Selon sa maturité psychosociale

- Selon son impulsivité

- Selon son statut socio-économique

- Selon sa santé mentale et psychologique

- Selon sa suggestibilité

- Autre:

10. Pensez-vous qu'il y ait des facteurs développementaux qui affectent l'aptitude des jeunes à subir un procès? $(1=$ non $/ 5=$ absolument / Je ne peux pas répondre) 
10a. Veuillez préciser quels sont les facteurs développementaux qui affectent l'aptitude des jeunes à subir un procès:

- L'immaturité psychosociale

- L'immaturité cognitive

- Le respect de l'autorité

- La vulnérabilité au stress

- Autre:

10b. Veuillez indiquer des suggestions concrètes à propos de la manière avec laquelle la compétence des jeunes devrait être déterminée:

11. Est-ce que l'application des sanctions punitives, comparativement à la réhabilitation, a un impact néfaste sur la santé mentale et psychologique des jeunes?

- Oui

- Non

- Je ne peux pas répondre

11a. Comment pourrait-on promouvoir la responsabilisation des jeunes envers leurs actes tout en respectant leur développement normal ainsi que leurs comportements positifs?

- Instaurer les programmes de réhabilitation à l'intérieur de la garde

- Procurer aux jeunes de l'aide professionnelle (ex.: psychologues, travailleurs sociaux) après qu'ils ont quitté la garde

- Garder les parents à proximité des jeunes

- Instaurer des programmes qui aideront les jeunes à s'intégrer dans un environnement sain

- Adapter les gardes/programmes de réhabilitation aux âges des jeunes

- Avoir des gardes/programmes de réhabilitation séparés pour les filles et les garçons

- Autre:

12. Qu'est-ce que la science développementale pourrait suggérer afin de contribuer au succès des programmes de réhabilitation des jeunes? - Impliquer les parents dans les programmes de réhabilitation

- Procurer un environnement sécuritaire

- Apprendre aux jeunes les habiletés sociales en les amenant coopérer dans des travaux d'équipe 
- Apprendre aux jeunes à contrôler leurs impulsions

- Apprendre aux jeunes à contrôler leurs émotions

- Apprendre aux jeunes à communiquer leurs émotions par des moyens socialement appropriés

- Promouvoir le développement moral des jeunes

- Créer des activités qui permettront aux jeunes de développer et de pratiquer la prise de décision ainsi que leur pensée critique

- Assister les jeunes dans l'établissement de buts personnels

- Sensibiliser les jeunes à propos de leurs forces et des contributions potentielles qu'ils pourraient effectuer

- Évaluer la santé psychologique des jeunes afin de mieux répondre à leurs besoins personnels

- Aider les jeunes à s'intégrer dans la société une fois qu'ils sont retirés du programme de garde ou de réhabilitation

- Je ne peux pas répondre

- Autre:

13. Pensez-vous qu'il devrait y avoir un âge minimum pour la responsabilité pénale des jeunes?

- Oui :

Quel devrait être l'âge minimum pour la responsabilité pénale des jeunes?

- Non

14. Pensez-vous qu'il devrait y avoir un âge minimum pour la responsabilité pénale des adultes?

- Oui :

Quel devrait être l'âge minimum pour la responsabilité pénale des adultes?

- Non 